\title{
The Unbiased Characteristic of Doppler Frequency in GNSS Antenna Array Processing
}

\author{
Yuchen Xie $\mathbb{D}$, Zhengrong Li $\mathbb{D}^{\mathbb{D}}$, Feiqiang Chen, Huaming Chen, and Feixue Wang \\ College of Electronic Science, National University of Defense Technology, Changsha 410073, China \\ Correspondence should be addressed to Yuchen Xie; olien_x@163.com and Zhengrong Li; zr_li@nudt.edu.cn
}

Received 10 December 2018; Accepted 7 March 2019; Published 24 April 2019

Academic Editor: Ikmo Park

Copyright (c) 2019 Yuchen Xie et al. This is an open access article distributed under the Creative Commons Attribution License, which permits unrestricted use, distribution, and reproduction in any medium, provided the original work is properly cited.

\begin{abstract}
The antenna array technology, especially the spaced-time array processing (STAP), is one of the effective methods used in Global Navigation Satellite System (GNSS) receivers to refrain the power of jamming and enhance the performance of receivers in the circumstance of interference. However, biases induced to the receiver because of many reasons, including characteristic of antennas, front-end channel electronics, and space-time filtering, are extremely harmful to the high precise positioning of receivers. Although plenty of works have been done to calibrate the antenna and to mitigate these biases, achieving a good performance of antijamming, high accuracy, and low complexity at the same time still remains challenging. Different from existing works, this paper leverages the characteristic of GNSS signal's Doppler frequency in STAP, which is proven to remain unbiased to solve the problem, even when the nonideal antennas are used and the interference circumstance changes. Since the integration of frequency is carrier phase, the unbiased Doppler frequency leads to an accurate estimation of carrier phase which can be used to calibrate the antenna array without extra apparatus or complicating algorithms. Therefore, a simple Doppler-aid strategy may be developed in the future to solve the difficulty of STAP bias mitigation.
\end{abstract}

\section{Introduction}

Array processing is one of the most effective ways to refrain the jamming aimed at GNSS receiver, and among those array processing methods, the use of controlled reception pattern antenna (CRPA) arrays has attracted more and more attention. CRPA arrays can provide beam forming/null steering in specific direction by adaptively adjusting the weight of each antenna element. Typically, an adaptive finite impulse response (FIR) filter placed behind each element allows a better performance of antijamming, which is known as the space-time array processing [1]. CRPA arrays especially STAP-based antenna arrays are so popular that numerous adaptive algorithms have been researched to provide a high performance of interference suppression [2].

Unfortunately, despite of the antijamming benefit that STAP can provide, undesirable and unpredictable biases are also induced to the GNSS receiver [2], which harms the accuracy of navigation solution [3-9]. There are several facts contributing to the STAP bias, including the overall gain and phase response of antennas, mutual coupling of antennas, front-end channel electronics, and space-time filtering. Previous research has focused on the code and carrier phase measurement bias using STAP. For space only processing (SOP), a code phase bias on the order of meters can be observed in simulation [3], and the phase bias of real data is discussed in [4]. The measurements are distorted further in STAP because of the applied FIR filters [5, 7]. In [1], the dependency of bias on interference circumstances is analyzed, which leads to the unpredictable characteristic of STAP-based bias.

Therefore, to fulfill the ability of adaptive arrays, researchers have made great efforts to mitigate these biases. One solution is to use an adaptive antenna array that exhibits small biases [8], or to do the precalibration to correct the antenna based bias, but it may be impractical to some low cost receivers $[10,11]$. Another way is to apply some specific algorithms that constrain the distortion of signal phase, but this method causes the loss of antijamming freedom degree. Some software based algorithms are also proposed to 
calibrate the bias $[12,13]$, but the computational complexity prevents them from the real time application. It seems that high accuracy, low complexity, and real time response are incompatible in the bias mitigation of STAP.

Different from previous works, this paper analyzes the performance of Doppler frequency during the processing of antenna arrays. Logically, neither the response of antennas and channels nor the adaptive weights will change the carrier frequency of signal. In this paper, the unbiased characteristic of Doppler frequency of GNSS signal in STAP is proved by using the auto correlation function (ACF), and the simulation results also show that the changing of interference leads to the uncertain variation of phase, but it will not affect the estimation of Doppler frequency of receiver. When the response of channel is not ideal, the unpredictable distortion of phase is more obvious, while the estimation of Doppler frequency is still unbiased. Since the integration of frequency is carrier phase, the unbiased characteristic of Doppler frequency can be critical to high precision GNSS applications. Although the deduction is based on the GNSS signal, the unbiased characteristic is common in array processing. It means that we can correct the phase error induced by STAP or calibrate the antenna instantaneously using the Doppler frequency without any extra complexity of algorithm or hardware.

The rest of this paper is organized as follows. First the array model is established and a brief description of the antenna induced bias in phase is given in Section 2. Then the unbiased characteristic of the Doppler frequency of GNSS signal is proved in Section 3 with the help of ACF. In Section 4, simulation results are presented to compare the phase error and the frequency error, which further proves the unbiased characteristic of Doppler frequency. Finally, the conclusion is made in Section 5.

\section{Array Model}

Although STAP is an effective way to mitigate the interference in GNSS signal [14], biases induced by antennas and algorithms are not negligible for precision applications, especially when a large antenna array with complicated filtering is considered [3]. This section presents a model of array processing for describing errors induced by STAP and proving their dependency on interference circumstances.

The antenna model is depicted in Figure 1. In this model, $\mathrm{K}$ individual antenna element with an M-tap FIR filter following is considered. $A_{k}(f, \theta, \varphi)$ represents the system response of the $k$ th element in $(\theta, \varphi)$ direction $(\theta$ and $\varphi$ stand for azimuth and elevation angles, respectively). $A_{k}$ is also affected by the frequency of received signals (the effect of mutual coupling is not considered). $F_{k}(f)$ stands for the electronic which downconverts the signal to baseband and perform analog-to-digital conversion. The output digital signal of each element is then filtered by an M-tap adaptive filter, and the frequency response of each FIR filter is denoted by $W_{k}(f)$. Then the outputs of the filters are summed for postprocessing.

The complex weight in STAP filter can be represented by a stack vector

$$
\mathbf{w}=\left[\begin{array}{c}
\mathbf{w}_{1} \\
\mathbf{w}_{2} \\
\vdots \\
\mathbf{w}_{K}
\end{array}\right]
$$

$\mathbf{w}_{k}$ is an $M \times 1$ vector corresponding to the $k$ th filter

$$
\mathbf{w}_{k}=\left[\begin{array}{llll}
w_{k 1} & w_{k 2} & \cdots & w_{k M}
\end{array}\right]^{\mathrm{T}}
$$

where $w_{k m}$ is each adaptive weight.

The instantaneous digital output snapshot on the taps of the $k$ th front-end channel is denoted by

$$
\mathbf{x}_{k}[n]=\left[\begin{array}{llll}
x_{k}[n] & x_{k}[n-1] & \cdots & x_{k}[n-M+1]
\end{array}\right]^{\mathrm{T}}
$$

$x_{k}[n]$ is the received signal on the $k$ th element, which contains desired signal $s_{k}[n]$, undesired interference signal $j_{k}[n]$, and noise $\eta_{k}[n]$.

$$
x_{k}[n]=s_{k}[n]+j_{k}[n]+\eta_{k}[n]
$$

The snapshot on each filter is combined into a received signal stack vector

$$
\mathbf{x}[n]=\left[\begin{array}{c}
\mathbf{x}_{1}[n] \\
\mathbf{x}_{2}[n] \\
\vdots \\
\mathbf{x}_{K}[n]
\end{array}\right]
$$

The STAP makes the weighted sum of signal vector by multiplying it with the weight vector

$$
y[n]=\mathbf{w}^{\mathrm{T}} \mathbf{x}[n]
$$

By applying some criterions [14], such as power minimization $[15,16]$, multiple constrained minimum variance [17], and minimum mean square error [18], the power of interference signal can be effectively restrained after the adaptive weighted summation.

However, characteristics of antennas and channels, as well as STAP algorithms, cause biases to the output signal. To measure them, it is reasonable to analyze their effects on the GNSS receiver cross-correlation.

The GNSS receiver correlators perform the crosscorrelation by multiplying local $\mathrm{C} / \mathrm{A}$ code replicas $r[n]$ with the received signal $s[n]$ and then doing the average of the correlation time $N_{r}[19]$.

$$
R(\tau)=\frac{1}{N_{r}} \sum_{n=1}^{N_{r}} s[n+\tau] r[n]
$$

$R(\tau)$, named as the auto correlation function, is the output of the correlator, where $\tau$ is the code delay between the received signal and the local replica.

In STAP, the correlation process can be treated as the correlation between the signal of each FIR filter tap and the 


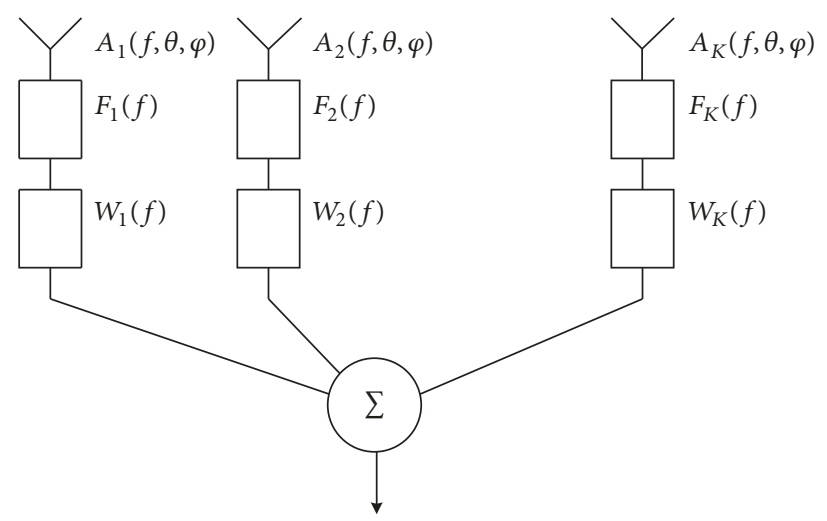

FIGURE 1: STAP-based adaptive antenna array model.

local C/A code replica, and then all of these $K \times M$ ACFs sum up after multiplying their weight.

$$
\begin{aligned}
R_{y d}(\tau)= & \frac{1}{N_{r}} \sum_{n=1}^{N_{r}} y[n+\tau] r[n] \\
= & \sum_{l=1}^{K M}\left|\boldsymbol{a}_{l}(f, \theta, \varphi)\right|\left|\boldsymbol{f}_{l}(f)\right|\left|w_{l}\right| R\left(\tau+\tau_{l}\right)+j^{\prime} \\
& +\eta^{\prime}
\end{aligned}
$$

$\left|\boldsymbol{a}_{l}(f, \theta, \varphi)\right|,\left|\boldsymbol{f}_{l}(f)\right|$ and $\left|w_{l}\right|$ are the amplitude effects of antenna receiving pattern, channel response, and adaptive weight, respectively, while $\tau_{l}$ is the total code delay induced by antenna, channel, and weight for each tap. $j^{\prime}$ and $\eta^{\prime}$ are the remaining interference and noise after correlation.

It can be known from (8) that the output ACF of STAP is a combination of $K \times M$ different ACFs whose amplitude and time delay vary from one to another. In fact, the antenna receiving pattern depends on the received signal's direction and frequency, the channel response changes with time and temperature, and adaptive weights are also affected by interference circumstance. Therefore, the bias induced by STAP in ACF changes with the GNSS signal, the interference, and the environment, which is consequently unpredictable.

Simulation results in different interference circumstances support this conclusion, which will be explained in detail in Section 4. In this case, even if STAP has successfully refrained the power of interferences, the navigation output which is based on code phase measuring and carrier phase aid may not be accurate. However, it can be proved that Doppler frequency is unbiased after array processing, which can be further used to enhance the measuring accuracy of receiver. The details of deduction will be presented in the next section.

\section{Doppler Frequency Estimation in STAP}

In the single array receiver, the $\mathrm{I} \backslash \mathrm{Q}$ orthogonal demodulation is applied to the received signal to move the carrier of it [19].
After orthogonal demodulation and correlation, the output of correlator can be denoted by

$$
\begin{aligned}
i[n] & =a D[n] R(\tau) \cos \left[2 \pi f_{e} n+\phi_{e}\right] \\
q[n] & =a D[n] R(\tau) \sin \left[2 \pi f_{e} n+\phi_{e}\right]
\end{aligned}
$$

$a$ is the amplitude of received signal and $D[n]$ is the data bit, both of which can be regarded as 1 for the sake of convenience. $R(\tau)$ is the ACF which has been defined in Section 2. $f_{e}$ and $\phi_{e}$ are the frequency and phase discrepancies between local carrier and received signal's carrier, respectively.

The relation among the local carrier $f_{\text {local }}$, the received signal's carrier $f_{\text {carr }}$, the frequency error $f_{e}$, the Doppler frequency of signal $f_{d}$, and the standard carrier frequency $f_{0}$ can be written as

$$
\begin{aligned}
f_{\text {carr }} & =f_{\text {local }}+f_{e} \\
f_{d} & =f_{\text {carr }}-f_{0}=f_{\text {local }}+f_{e}-f_{0}
\end{aligned}
$$

Ideally, $f_{e}$ is zero, the Doppler frequency will exactly be the discrepancy between local generated carrier frequency and the standard frequency, i.e., $f_{\text {local }}-f_{0}$. But in real situation $f_{e}$ is nonzero and includes the Doppler frequency estimation error and some other noise errors. Although they are difficult to be separated to get the exact Doppler frequency, in simulation test, with setting Doppler frequency and nonsignificant estimation error, the Doppler frequency can be reasonably estimated by calculating $f_{e}$.

Therefore, the $f_{e}$ calculating process of the GNSS receiver in its fine acquisition is firstly introduced. By simplifying (9) and (10) and doing the square, we get

$$
\begin{aligned}
i^{2}[n] & =R^{2}(\tau) \cos ^{2}\left[2 \pi f_{e} n+\phi_{e}\right] \\
q^{2}[n] & =R^{2}(\tau) \sin ^{2}\left[2 \pi f_{e} n+\phi_{e}\right]
\end{aligned}
$$

Further, we set

$$
\begin{aligned}
& z_{r}[n]=i^{2}[n]-q^{2}[n]=R^{2}(\tau) \cos \left[4 \pi f_{e} n+2 \phi_{e}\right] \\
& z_{i}[n]=2 i[n] q[n]=R^{2}(\tau) \sin \left[4 \pi f_{e} n+2 \phi_{e}\right]
\end{aligned}
$$


Combining $z_{r}[n]$ and $z_{i}[n]$ into a complex signal, we get

$$
\begin{aligned}
z[n]= & z_{r}[n]+j z_{i}[n] \\
= & R^{2}(\tau) \cos \left[4 \pi f_{e} n+2 \phi_{e}\right] \\
& +j R^{2}(\tau) \sin \left[4 \pi f_{e} n+2 \phi_{e}\right]
\end{aligned}
$$

It is noticed that $z[n]$ is a single frequency complex signal with the amplitude of $R^{2}(\tau)$, the frequency of $2 f_{e}$, and the phase of $2 \phi_{e}$. After doing the Fast Fourier Transform (FFT) to $z[n]$, the maximum in its frequency domain is located at $2 f_{e}$ which is unrelated to its phase error $2 \phi_{e}$.

Therefore, $f_{e}$ can be achieved by

$$
f_{e}=\frac{1}{2} \operatorname{findmax}(\operatorname{FFT}(z[n]))
$$

where findmax means searching for the frequency maximizing $|\operatorname{FFT}(z[n])|$.

In the STAP receiver, the $\mathrm{I} \backslash \mathrm{Q}$ orthogonal demodulation is also applied to the received signal, and derived from (8), (9), and (10), it can be rewritten as

$$
\begin{aligned}
i_{\text {array }}[n] & =\sum_{l=1}^{K M} b_{l} R\left(\tau+\tau_{l}\right) \cos \left[2 \pi f_{e} n+\phi_{e}+\phi_{l}\right] \\
q_{\text {array }}[n] & =\sum_{l=1}^{K M} b_{l} R\left(\tau+\tau_{l}\right) \sin \left[2 \pi f_{e} n+\phi_{e}+\phi_{l}\right]
\end{aligned}
$$

where

$$
b_{l}=\left|\boldsymbol{a}_{l}(f, \theta, \varphi)\right|\left|\boldsymbol{f}_{l}(f)\right|\left|w_{l}\right|
$$

is the coefficient containing all amplitude effects. $f_{e}$ has been defined in (10), and jammer induced Doppler shift at the receiver is assumed to be small compared to satellite Doppler. $\tau_{l}$ has been defined in (8), and $\phi_{l}$ is the total carrier phase error induced by STAP. As mentioned before, $b_{l}, \tau_{l}$, and $\phi_{l}$ vary from one to another.

Similarly, the square and multiplication of (19) and (20) are

$$
\begin{gathered}
i_{\text {array }}^{2}[n]=\sum_{l=1}^{K M} \sum_{p=1}^{K M}\left\{\begin{array}{c}
b_{l} b_{p} R\left(\tau+\tau_{l}\right) R\left(\tau+\tau_{p}\right) \cdot \\
\cos \left[2 \pi f_{e} n+\phi_{e}+\phi_{l}\right] \cdot \\
\cos \left[2 \pi f_{e} n+\phi_{e}+\phi_{p}\right]
\end{array}\right\} \\
q_{\text {array }}^{2}[n]=\sum_{l=1}^{K M} \sum_{p=1}^{K M}\left\{\begin{array}{c}
b_{l} b_{p} R\left(\tau+\tau_{l}\right) R\left(\tau+\tau_{p}\right) \cdot \\
\sin \left[2 \pi f_{e} n+\phi_{e}+\phi_{l}\right] \cdot \\
\sin \left[2 \pi f_{e} n+\phi_{e}+\phi_{p}\right]
\end{array}\right\} \\
i_{\text {array }} q_{\text {array }}[n] \\
=\sum_{l=1}^{K M} \sum_{p=1}^{K M}\left\{\begin{array}{c}
b_{l} b_{p} R\left(\tau+\tau_{l}\right) R\left(\tau+\tau_{p}\right) \cdot \\
\cos \left[2 \pi f_{e} n+\phi_{e}+\phi_{l}\right] \cdot \\
\sin \left[2 \pi f_{e} n+\phi_{e}+\phi_{p}\right]
\end{array}\right\}
\end{gathered}
$$

So $z_{\text {rarray }}[n]$ and $z_{\text {i-array }}[n]$ can be denoted as

$$
\begin{aligned}
z_{\text {rarray }}[n] & =i_{\text {array }}^{2}[n]-q_{\text {array }}^{2}[n] \\
& =\sum_{l=1}^{K M K M} \sum_{p=1}^{K M}\left\{\begin{array}{c}
b_{l} b_{p} R\left(\tau+\tau_{l}\right) R\left(\tau+\tau_{p}\right) \cdot \\
\cos \left[4 \pi f_{e} n+2 \phi_{e}+\phi_{l}+\phi_{p}\right]
\end{array}\right\} \\
z_{\text {iarray }}[n] & =2 i_{\text {array }}[n] q_{\text {array }}[n] \\
& =\sum_{l=1}^{K M} \sum_{p=1}^{K M}\left\{\begin{array}{c}
b_{l} b_{p} R\left(\tau+\tau_{l}\right) R\left(\tau+\tau_{p}\right) \cdot \\
\sin \left[4 \pi f_{e} n+2 \phi_{e}+\phi_{l}+\phi_{p}\right]
\end{array}\right\}
\end{aligned}
$$

and

$$
\begin{aligned}
& z_{\text {array }}[n]=z_{\text {r_array }}[n]+j z_{i_{-a r r a y}}[n] \\
& =\sum_{l=1}^{K M} \sum_{p=1}^{K M}\left\{\begin{array}{c}
b_{l} b_{p} R\left(\tau+\tau_{l}\right) R\left(\tau+\tau_{p}\right) \cdot \\
\cos \left[4 \pi f_{e} n+2 \phi_{e}+\phi_{l}+\phi_{p}\right]
\end{array}\right\} \\
& \quad+j \sum_{i=1}^{K M} \sum_{j=1}^{K M}\left\{\begin{array}{c}
b_{l} b_{p} R\left(\tau+\tau_{l}\right) R\left(\tau+\tau_{p}\right) \cdot \\
\sin \left[4 \pi f_{e} n+2 \phi_{e}+\phi_{l}+\phi_{p}\right]
\end{array}\right\}
\end{aligned}
$$

Therefore, $z_{\text {array }}[n]$ is the sum of $K M \times K M$ single frequency complex signals. Although they are different in amplitude and carrier phase, as

$$
\begin{aligned}
\left|z_{\text {array }\lrcorner_{p}}[n]\right| & =b_{l} b_{p} R\left(\tau+\tau_{l}\right) R\left(\tau+\tau_{p}\right) \\
\phi_{\text {array }\lrcorner p} & =2 \phi_{e}+\phi_{l}+\phi_{p}
\end{aligned}
$$

they have the same frequency $2 f_{e}$. In this case, (18) is still effective in estimating the Doppler frequency.

Based on these deductions, it is reasonable to say that unlike code and carrier phase which will be unpredictably shifted because of the changing of interferences, the Doppler frequency of the received signal remains unbiased in array processing.

In fact, even in the situation that the bias of phase is too severe to calibrate, or in the situation that the electronic characteristic of analog element changes, which makes the previous calibration ineffective, the Doppler frequency still remains unbiased because interference and STAP only affect the received signal's phase rather than its frequency.

Although the deduction is based on GNSS signal, it is also true to other signals of array processing. As the integration of frequency through time leads to phase, the phase error can also be corrected with the help of Doppler frequency. Therefore, the unbiased and accurate Doppler frequency is especially useful to high precision locating applications. In another way, the integration of the Doppler frequency can also be used to calibrate the bias induced by STAP, as the difference between the integration Doppler frequency and the output carrier phase is the total bias of STAP. In that case, the real time calibration for STAP can be realized to significantly enhance the performance of array processing. 
TABLE 1: Simulation parameters.

\begin{tabular}{lc}
\hline Parameter & Value \\
\hline Signal Type & BeiDou \\
Carrier Frequency & $1268.52 \mathrm{MHz}$ \\
Signal Length & $1000 \mathrm{~ms}$ \\
Intermediate Frequency & $46.52 \mathrm{MHz}$ \\
Sampling Frequency & $61 \mathrm{MHz}$ \\
Code Frequency & $10.23 \mathrm{MHz}$ \\
Code Length & 10230 \\
Signal Noise Ratio (SNR) & $-15 \mathrm{~dB}$ \\
Signal Direction & $\left(\theta=85^{\circ}, \varphi=70^{\circ}\right)$ \\
Jamming Noise Ratio (JNR) & Jammerl: $50 \mathrm{~dB}$, Jammer2: $30 \mathrm{~dB}$ \\
Antenna Element & 4 \\
Array Structure & Circular \\
Time Taps & $3,5,7$ \\
Anti Jamming Criterion & PI \\
\hline
\end{tabular}

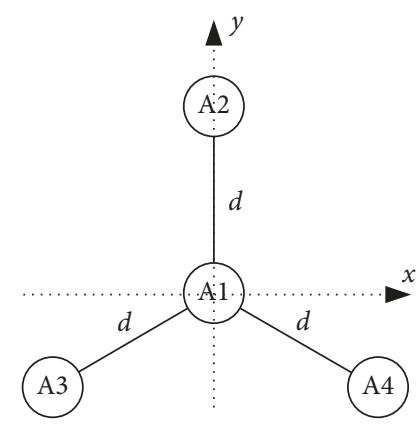

Figure 2: Structure of antenna array.

Simulation results in Section 4 support the conclusion above and further prove the unbiased characteristic of Doppler frequency.

\section{Simulation Results}

In this section, simulations of the typical BeiDou receiver's performance in different interference circumstances are presented to analyze the bias induced by STAP.

The simulated receiver is a 4-element circular antenna array receiver, which has one antenna at the origin point with three others surrounded, and the distance $d$ from the origin to each other antenna is half carrier wavelength. The structure of the antenna array is shown in Figure 2.

The STAP with an FIR filter back to each antenna is applied, and the power inverse (PI) [20] criterion is chosen to adapt the weight of each tap, which minimizes the output of antenna array processing to mitigate the effect of jammer. Meanwhile, the same signal received in an interference free circumstance and without applying any antijamming method is also processed by the receiver as the reference. The parameters for this simulation are listed in Table 1.

In the simulation, the circumstance of interferences changes with time and contains different types, directions, and powers of jamming. The simulation includes four steps as follows.

Step 1. Turn on the signal $\left(\theta=85^{\circ}, \varphi=70^{\circ}\right.$, SNR: $\left.-15 \mathrm{~dB}\right)$.

Step 2. Turn on the jammerl $\left(\theta=300^{\circ}, \varphi=5^{\circ}\right.$, JNR: $\left.50 \mathrm{~dB}\right)$ at the $200 \mathrm{~ms}$.

Step 3. Turn on the jammer2 $\left(\theta=135^{\circ}, \varphi=10^{\circ}\right.$, JNR: $\left.30 \mathrm{~dB}\right)$ at the $400 \mathrm{~ms}$.

Step 4. Change the direction of jammerl $\left(\theta=180^{\circ}, \varphi=30^{\circ}\right)$ at the $600 \mathrm{~ms}$.

Step 5. Turn off the jammerl and the jammer2 at the $800 \mathrm{~ms}$.

The jammerl emits a white noise interference with the band of $20.46 \mathrm{MHz}$ centered at $1268.52 \mathrm{MHz}$, while the jammer2 emits a single frequency interference closed to the signal's frequency. During the whole time of simulation, the signal is combined with white noise.

4.1. Ideal Channel Simulation. In the first simulation, we focus on the bias induced by adaptive algorithm; therefore ideal antennas and channels are considered. Figures 3 and 4 show the simulation results. 


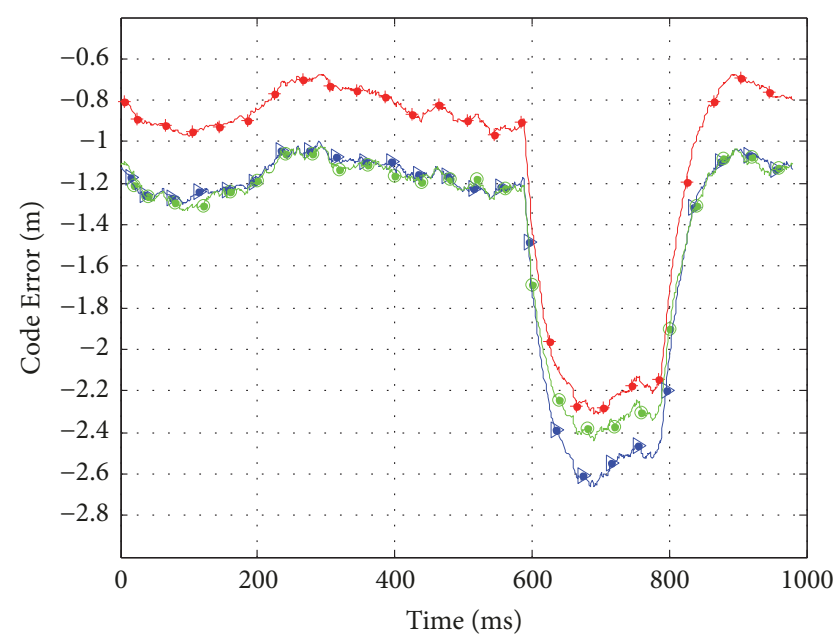

$\begin{array}{rr}\perp & \text { 3Taps } \\ \square & \text { 5Taps } \\ - & \text { 7Taps }\end{array}$

(a) Code phase error in simulation1

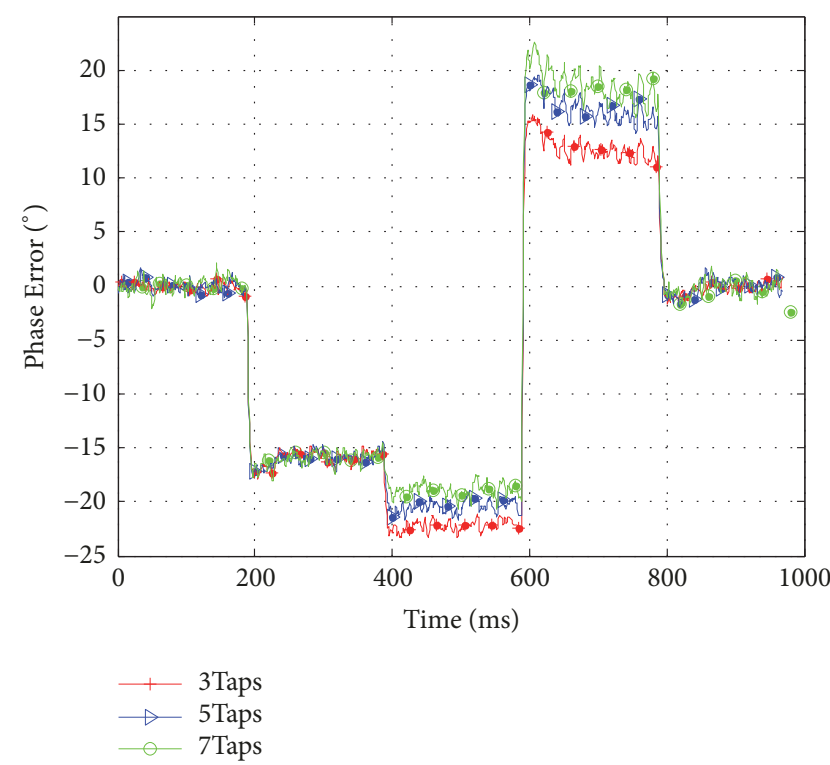

(b) Carrier phase error in simulation1

Figure 3

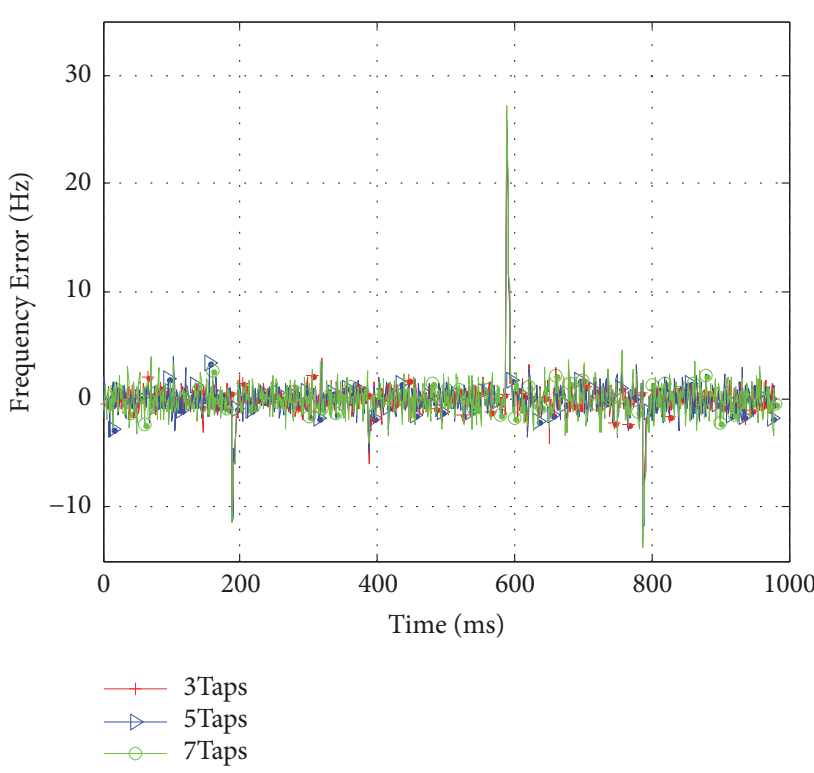

(a) Tracking loop frequency error in simulationl

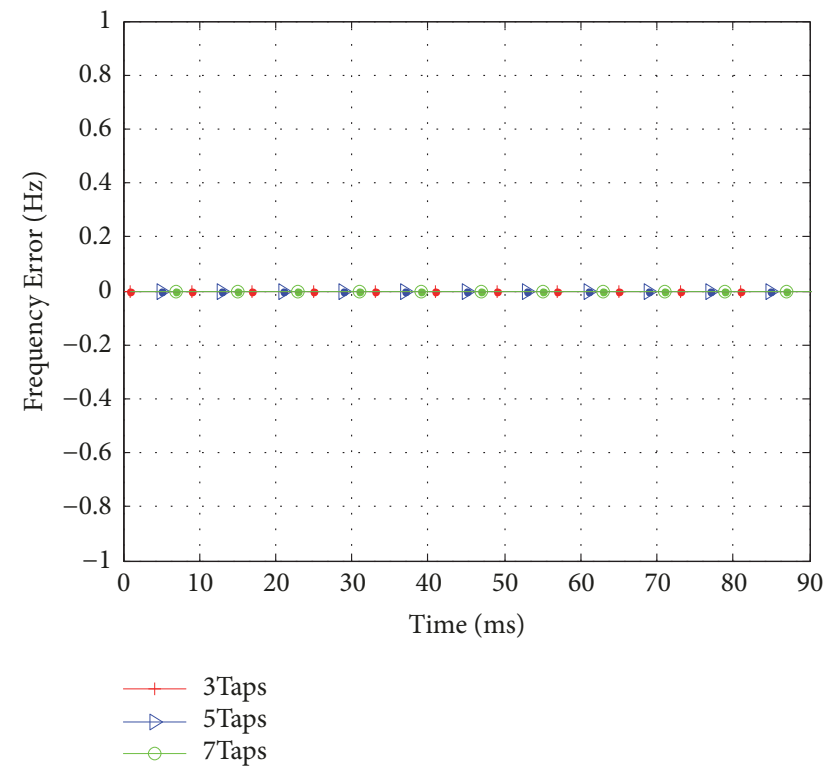

(b) Fine acquisition error in simulation1

FigURE 4

Figure 3(a) is the difference of code phase between the signal after STAP and the reference signal, and Figure 3(b) is its counterpart carrier phase difference. It can be known from the figures that STAP successfully restrains the power of interferences and enables the receiver to keep tracking of the code phase. Nevertheless, even when the jammers are turned off, the code error is not zero because STAP induces bias to the receiver. Taking the 3-tap filter simulation in Figure 3(a) as example, the average error is $-0.907 \mathrm{~m}$ ( $1 \mathrm{~ms}$ to $200 \mathrm{~ms}$ ) with the minimum of $0.799 \mathrm{~m}$ at the $1 \mathrm{~ms}$, considering that one chip corresponds to $30 \mathrm{~m}$ in our simulation. When the jammerl is turned on and switched, the code phase error varies slightly, as the average error is $-0.744 \mathrm{~m}$ during $200 \mathrm{~ms}$ to $400 \mathrm{~ms}$ and $-0.904 \mathrm{~m}$ during $400 \mathrm{~ms}$ to $600 \mathrm{~ms}$. However, when the jammer2 is turned on as well, the error of code phase sees a dramatic jump near the $600 \mathrm{~ms}$, after which it fluctuates severely, and the average error is $-2.124 \mathrm{~m}$ during $600 \mathrm{~ms}$ to $800 \mathrm{~ms}$. The situations for 5- and 7-tap filter are similar but the errors are more severe than that of 3-tap filter.

As for the carrier phase error, it can be known from Figure 3(b) that it strongly depends on the circumstance of interference. In detail, when there is no interference, the 
TABLE 2

(a) Average phase error in simulationl

\begin{tabular}{llccccc}
\hline $\begin{array}{l}\text { err } \\
\text { erded }\end{array}$ & $0-200 \mathrm{~ms}$ & $200-400 \mathrm{~ms}$ & $400-600 \mathrm{~ms}$ & $600-800 \mathrm{~ms}$ & $800-1000 \mathrm{~ms}$ & total \\
\hline \multirow{2}{*}{3 taps } & $-0.907 \mathrm{~m} /$ & $-0.744 \mathrm{~m} /$ & $-0.904 \mathrm{~m} /$ & $-\mathbf{2 . 1 2 4 m} /$ & $-0.877 \mathrm{~m} /$ & $-1.114 \mathrm{~m} /$ \\
& $-0.936^{\circ}$ & $-\mathbf{1 6 . 2 2 7 ^ { \circ }}$ & $-20.362^{\circ}$ & $12.110^{\circ}$ & $-0.266^{\circ}$ & $-5.195^{\circ}$ \\
\multirow{3}{*}{5 taps } & $-1.233 \mathrm{~m} /$ & $-1.081 \mathrm{~m} /$ & $-1.210 \mathrm{~m} /$ & $-\mathbf{2 . 4 4 6 m} /$ & $-1.211 \mathrm{~m} /$ & $-1.439 \mathrm{~m} /$ \\
& $-0.864^{\circ}$ & $-16.199^{\circ}$ & $-18.508^{\circ}$ & $15.585^{\circ}$ & $-0.094^{\circ}$ & $-4.064^{\circ}$ \\
7 taps & $-1.247 \mathrm{~m} /$ & $-1.098 \mathrm{~m} /$ & $-1.216 \mathrm{~m} /$ & $\mathbf{- 2 . 2 6 4 m} /$ & $-1.214 \mathrm{~m} /$ & $-1.410 \mathrm{~m} /$ \\
& $-0.773^{\circ}$ & $-16.135^{\circ}$ & $-16.948^{\circ}$ & $\mathbf{1 7 . 6 8 2 ^ { \circ }}$ & $-0.290^{\circ}$ & $-3.329^{\circ}$ \\
\hline
\end{tabular}

(b) Standard deviation of phase in simulationl

\begin{tabular}{|c|c|c|c|c|c|c|}
\hline $\begin{array}{l}\sigma_{\text {code }} \\
\rho_{\text {carr }}\end{array}$ & $0-200 \mathrm{~ms}$ & $200-400 \mathrm{~ms}$ & $400-600 \mathrm{~ms}$ & $600-800 \mathrm{~ms}$ & $800-1000 \mathrm{~ms}$ & total \\
\hline 3 taps & $\begin{array}{c}0.041 \mathrm{~m} / \\
0.382^{\circ}\end{array}$ & $\begin{array}{c}0.041 \mathrm{~m} / \\
0.650^{\circ}\end{array}$ & $\begin{array}{c}0.088 \mathrm{~m} / \\
0.479^{\circ}\end{array}$ & $\begin{array}{c}0.188 \mathrm{~m} / \\
\mathbf{1 . 0 8 0}^{\circ}\end{array}$ & $\begin{array}{c}\mathbf{0 . 2 4 5 m} / \\
0.550^{\circ}\end{array}$ & $\begin{array}{c}0.532 \mathrm{~m} / \\
12.527^{\circ}\end{array}$ \\
\hline 5 taps & $\begin{array}{c}0.036 \mathrm{~m} / \\
0.507^{\circ}\end{array}$ & $\begin{array}{c}0.036 \mathrm{~m} / \\
0.589^{\circ}\end{array}$ & $\begin{array}{c}0.086 \mathrm{~m} / \\
0.575^{\circ}\end{array}$ & $\begin{array}{c}0.200 \mathrm{~m} / \\
1.270^{\circ}\end{array}$ & $\begin{array}{c}\mathbf{0 . 2 2 8 m} / \\
0.714^{\circ}\end{array}$ & $\begin{array}{c}0.530 \mathrm{~m} / \\
13.193^{\circ}\end{array}$ \\
\hline 7 taps & $\begin{array}{c}0.056 \mathrm{~m} / \\
0.660^{\circ}\end{array}$ & $\begin{array}{c}0.040 \mathrm{~m} / \\
0.667^{\circ}\end{array}$ & $\begin{array}{c}0.078 \mathrm{~m} / \\
0.610^{\circ}\end{array}$ & $\begin{array}{c}0.159 \mathrm{~m} / \\
\mathbf{1 . 4 9 9}^{\circ}\end{array}$ & $\begin{array}{c}\mathbf{0 . 2 0 0 m} / \\
0.817^{\circ}\end{array}$ & $\begin{array}{c}0.450 \mathrm{~m} / \\
13.543^{\circ}\end{array}$ \\
\hline
\end{tabular}

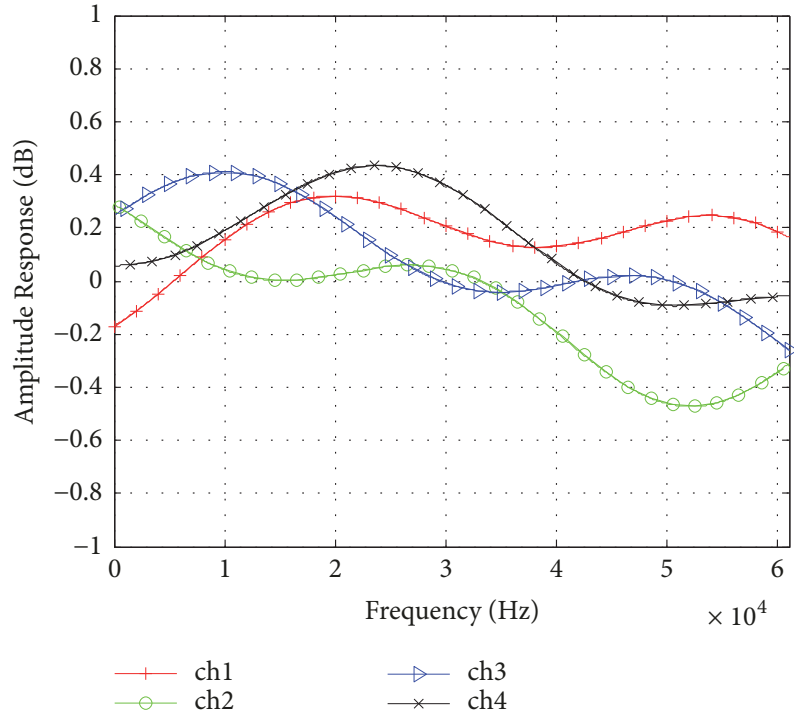

(a) Channel amplitude response

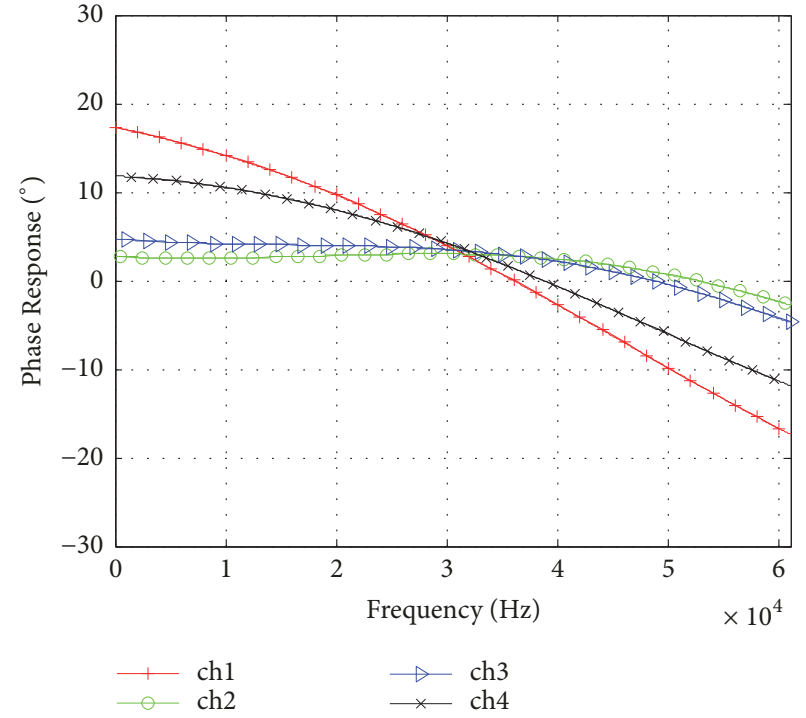

(b) Channel phase response

Figure 5

carrier phase error fluctuates around zero in the 3-tap filter simulation, with a maximal absolute value of $1.003^{\circ}$ at the $131 \mathrm{~ms}$. However, when turning on the jammers or switching the direction of jammerl, the carrier phase error jumps dramatically, as can be seen at the $200 \mathrm{~ms}, 400 \mathrm{~ms}, 600 \mathrm{~ms}$, and $800 \mathrm{~ms}$. Besides, when there exist interferences, the average of error is $-16.227^{\circ}$ (200ms to $\left.400 \mathrm{~ms}\right),-20.362^{\circ}$ ( $400 \mathrm{~ms}$ to $600 \mathrm{~ms}$ ), and $12.110^{\circ}(600 \mathrm{~ms}$ to $800 \mathrm{~ms})$, respectively, which presents an obvious bias from zero. It can also be noticed that the error fluctuates more drastically during the period from $600 \mathrm{~ms}$ to $800 \mathrm{~ms}$ when interferences are more complicated. The number of filter taps also influences the phase error but not significantly as can be seen from $400 \mathrm{~ms}$ to $600 \mathrm{~ms}$ in Figure 3(b).
The average of code phase error and carrier phase error (denoted as $e r r_{\text {code }}$ and $e r r_{\text {carr }}$ ), as well as their standard deviation (denoted as $\sigma_{\text {code }}$ and $\sigma_{\text {carr }}$ ), is shown in Tables 2(a) and 2(b) with the maximal value of each row being bold.

Figures 4(a) and 4(b) present two types of Doppler frequency errors in the simulation using different estimation methods. Data in Figure 4(a) is calculated from the output carrier frequency of the tracking loop while, in Figure 4(b), fine acquisition is applied to each $10 \mathrm{~ms}$ signal to estimate the accurate Doppler frequency. It can be noticed in Figure 4(a) that as the tracking loop calculates the frequency by using carrier phase, when the interference changes, which causes dramatic jumps to the code phase at $200 \mathrm{~ms}, 400 \mathrm{~ms}, 600 \mathrm{~ms}$, and $800 \mathrm{~ms}$, the output frequency jumps consequently. However, 
TABLE 3

(a) Average phase error in simulation2

\begin{tabular}{|c|c|c|c|c|c|c|}
\hline $\begin{array}{l}e r r_{\text {codel }} / \\
\text { err } \text { carr }\end{array}$ & $0-200 \mathrm{~ms}$ & $200-400 \mathrm{~ms}$ & $400-600 \mathrm{~ms}$ & $600-800 \mathrm{~ms}$ & $800-1000 \mathrm{~ms}$ & total \\
\hline 3 taps & $\begin{array}{c}0.242 \mathrm{~m} / \\
96.960^{\circ}\end{array}$ & $\begin{array}{c}1.803 \mathrm{~m} / \\
\mathbf{- 1 5 9 . 0 3 5 ^ { \circ }}\end{array}$ & $\begin{array}{l}1.645 \mathrm{~m} / \\
-156.356^{\circ}\end{array}$ & $\begin{array}{c}3.210 \mathrm{~m} / \\
-83.518^{\circ}\end{array}$ & $\begin{array}{c}1.029 \mathrm{~m} / \\
1.545^{\circ}\end{array}$ & $\begin{array}{l}1.593 \mathrm{~m} / \\
-60.829^{\circ}\end{array}$ \\
\hline 5 taps & $\begin{array}{c}0.108 \mathrm{~m} / \\
68.139^{\circ}\end{array}$ & $\begin{array}{c}0.901 \mathrm{~m} / \\
\mathbf{- 1 6 0 . 0 1 9 ^ { \circ }}\end{array}$ & $\begin{array}{c}0.817 \mathrm{~m} / \\
-158.376^{\circ}\end{array}$ & $\begin{array}{r}3.016 \mathrm{~m} / \\
-91.127^{\circ}\end{array}$ & $\begin{array}{c}2.247 \mathrm{~m} / \\
16.234^{\circ}\end{array}$ & $\begin{array}{r}1.408 \mathrm{~m} / \\
-66.017^{\circ}\end{array}$ \\
\hline 7 taps & $\begin{array}{c}0.126 \mathrm{~m} / \\
32.475^{\circ}\end{array}$ & 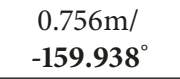 & $\begin{array}{l}0.706 \mathrm{~m} / \\
-158.293^{\circ}\end{array}$ & $\begin{array}{l}2.541 \mathrm{~m} / \\
-94.550^{\circ}\end{array}$ & $\begin{array}{r}1.805 \mathrm{~m} / \\
46.786^{\circ}\end{array}$ & $\begin{array}{l}1.179 \mathrm{~m} / \\
-68.082^{\circ}\end{array}$ \\
\hline
\end{tabular}

(b) Standard deviation of phase in simulation2

\begin{tabular}{|c|c|c|c|c|c|c|}
\hline 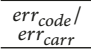 & $0-200 \mathrm{~ms}$ & $200-400 \mathrm{~ms}$ & $400-600 \mathrm{~ms}$ & $600-800 \mathrm{~ms}$ & $800-1000 \mathrm{~ms}$ & total \\
\hline 3 taps & $\begin{array}{l}1.062 \mathrm{~m} / \\
\mathbf{1 4 6 . 9 4 1}^{\circ}\end{array}$ & $\begin{array}{c}0.407 \mathrm{~m} / \\
0.609^{\circ}\end{array}$ & $\begin{array}{c}0.096 \mathrm{~m} / \\
14.355^{\circ}\end{array}$ & $\begin{array}{c}0.455 \mathrm{~m} / \\
19.584^{\circ}\end{array}$ & $\begin{array}{c}2.183 \mathrm{~m} / \\
2.151^{\circ}\end{array}$ & $\begin{array}{l}1.593 \mathrm{~m} / \\
119.025^{\circ}\end{array}$ \\
\hline 5 taps & $\begin{array}{l}1.170 \mathrm{~m} / \\
162.459^{\circ}\end{array}$ & $\begin{array}{c}0.260 \mathrm{~m} / \\
0.723^{\circ}\end{array}$ & $\begin{array}{c}0.080 \mathrm{~m} / \\
13.630^{\circ}\end{array}$ & $\begin{array}{l}0.629 \mathrm{~m} / \\
134.521^{\circ}\end{array}$ & $\begin{array}{l}1.908 \mathrm{~m} / \\
178.308^{\circ}\end{array}$ & $\begin{array}{l}1.478 \mathrm{~m} / \\
142.145^{\circ}\end{array}$ \\
\hline 7 taps & $\begin{array}{l}1.193 \mathrm{~m} / \\
\mathbf{1 7 3 . 1 8 6}^{\circ}\end{array}$ & $\begin{array}{c}0.227 \mathrm{~m} / \\
0.711^{\circ}\end{array}$ & $\begin{array}{c}0.079 \mathrm{~m} / \\
13.246^{\circ}\end{array}$ & $\begin{array}{l}0.552 \mathrm{~m} / \\
34.942^{\circ}\end{array}$ & $\begin{array}{l}2.121 \mathrm{~m} / \\
172.617^{\circ}\end{array}$ & $\begin{array}{l}1.403 \mathrm{~m} / \\
141.533^{\circ}\end{array}$ \\
\hline
\end{tabular}

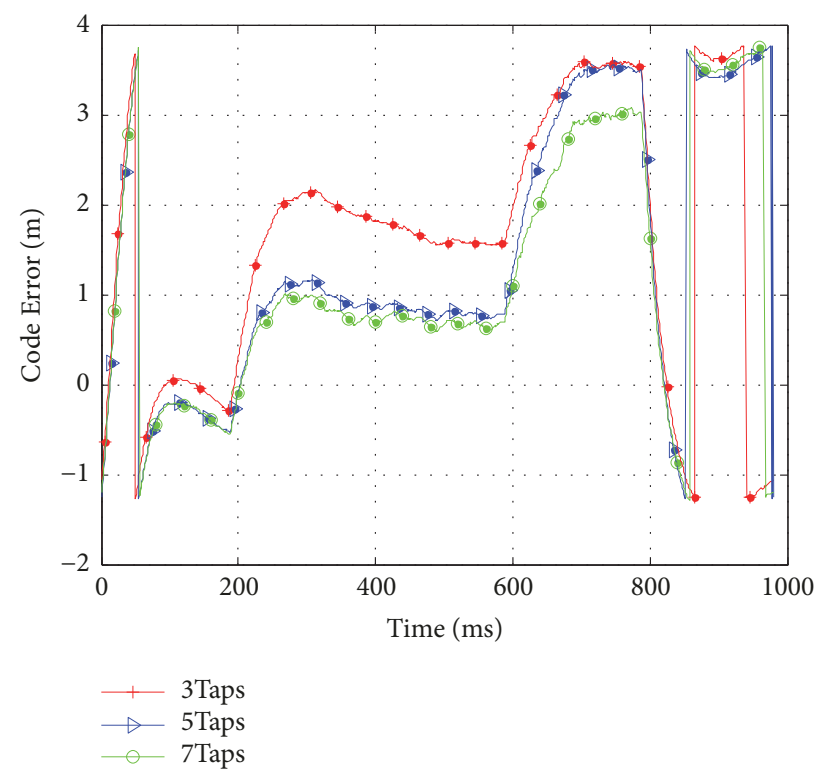

(a) Code phase error in simulation2

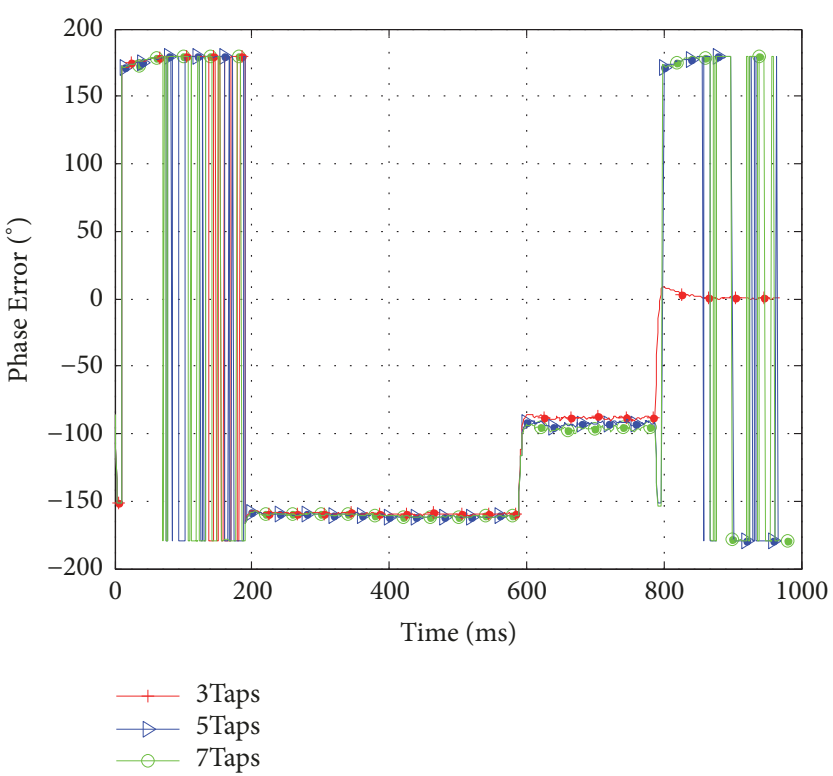

(b) Carrier phase error in simulation2

Figure 6

after this variation, the output frequency returns back to its original value and the error fluctuates around zero; for example, the average error is $-0.002 \mathrm{~Hz}$ for 3-tap filter simulation. This can be further proved in Figure 4(b) where the accurate Doppler frequency is estimated; the errors are exact zero for different taps filter simulations during the whole simulation time.

4.2. Imperfect Channel Simulation. In the second simulation, imperfect antennas and channels are taken into consideration. The characteristics of channels are presented in Figures 5(a) and 5(b), whose amplitude response waves randomly rang from $-0.5 \mathrm{~dB}$ to $0.5 \mathrm{~dB}$ and phase response is nonlinear with a maximal shift of $30^{\circ}$. The other parameters and steps are exactly the same as those in the first simulation, and the results are shown in Figures 5-7.

Comparing Figure 6(a) with Figure 3(a), it is obvious that the nonideal response of channels worsens the error of phase to vary more randomly; the gap between the maximum and minimum errors is about $5.039 \mathrm{~m}$. Similarly, the comparison between Figures 6(b) and 3(b) also shows a more drastic and random variation of the carrier phase, and the stable states of two pictures are different as well, which suggests new biases are induced because of channel characteristics.

err code , err carr,$\sigma_{\text {code }}$, and $\sigma_{\text {carr }}$ of the second simulation are shown in Table 3. 


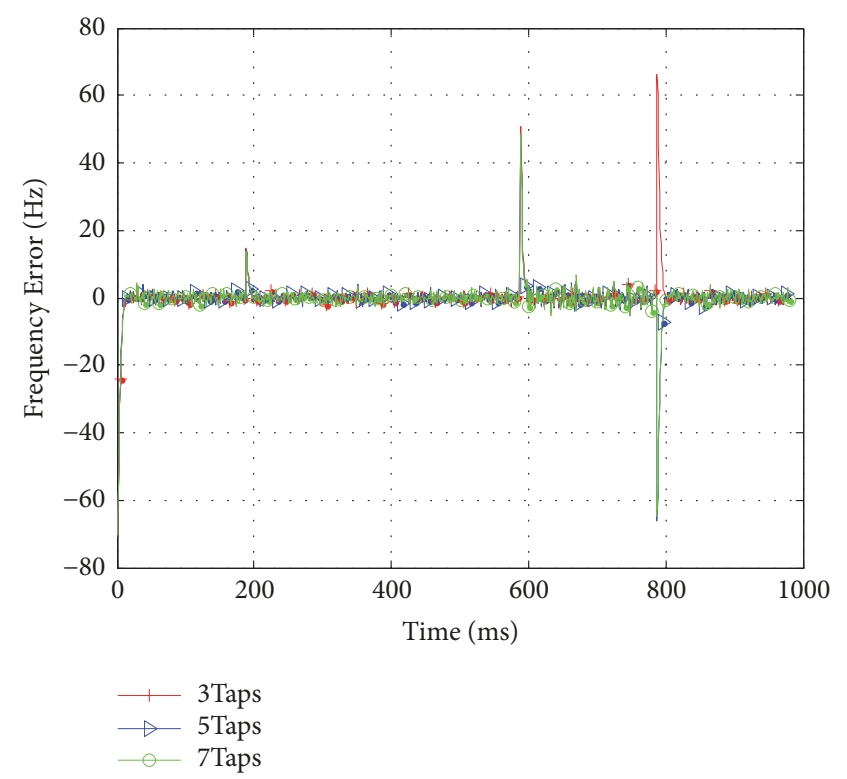

(a) Tracking loop frequency error in simulation2

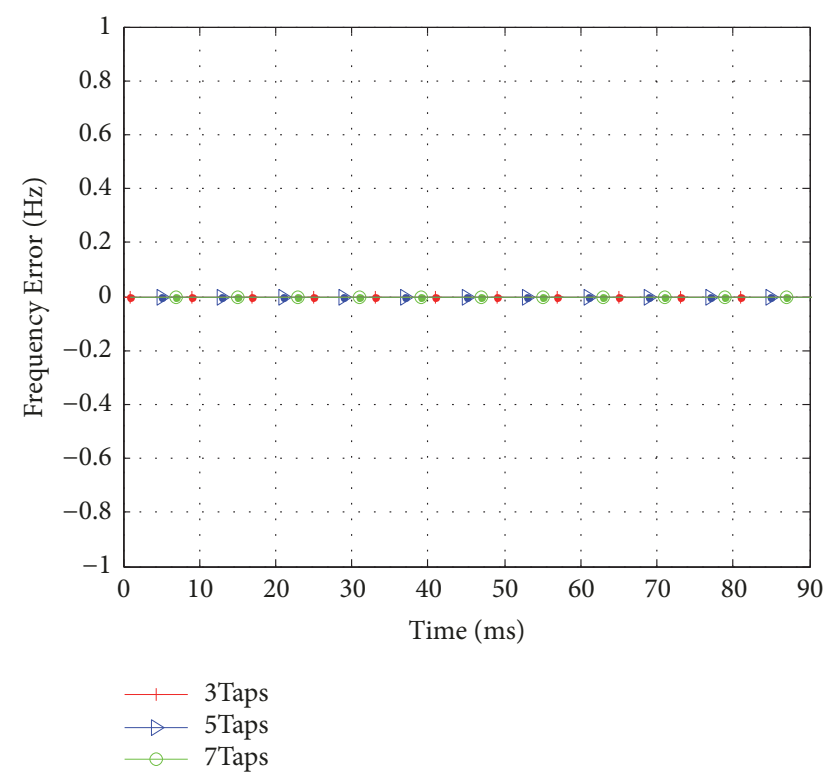

(b) Fine acquisition error in simulation2

Figure 7

On the contrary, it can be figured out in Figures 7(a) and 7(b) that the frequency error remains unbiased even with the consideration of channel effect. Although the deviation in Figure 7(a) is larger than that in Figure 4(a), the error returns to fluctuate around zero very soon, and the fine acquisition result in Figure 7(b) is the same zero as that in Figure 4(b).

Based on the simulation results above, it can be concluded that STAP induces unpredictable bias into receivers, which causes errors in the estimation of code and carrier phase, and the situation is even worse when antennas and channels are nonideal. However, thanks to the unbiased characteristic of Doppler frequency, the estimation of frequency in our simulation remains stable no matter how the interference circumstance changes.

\section{Conclusion}

This paper analyzes the bias induced by STAP of the phase of the GNSS antenna array receiver and proves the unbiased characteristic of Doppler frequency of it. Simulation results show that the distortion of phase is unpredictable, and it will be even worse when the nonideal antennas are used or the interference circumstance changes. On the contrary, the Doppler frequency remains unbiased in these situations, which can be used to estimate an unbiased carrier phase to enhance the accuracy of positioning. Since a goodperformance, low-complexity, and real-time bias mitigation is difficult to be realized by traditional methods, the Doppleraid carrier phase correction may be a simple and effective way to achieve this goal.

\section{Data Availability}

The data used to support the findings of this study are included within the article.

\section{Conflicts of Interest}

The authors declare that there are no conflicts of interest regarding the publication of this paper.

\section{Acknowledgments}

This work is supported by the National Natural Science Foundation of China under Grant no. 41604016.

\section{References}

[1] Z. Lu, J. Nie, F. Chen, and G. Ou, "Impact on antijamming performance of channel mismatch in GNSS antenna arrays receivers," International Journal of Antennas and Propagation, vol. 2016, Article ID 1909708, 9 pages, 2016.

[2] T. Marathe, S. Daneshmand, and G. Lachapelle, "Assessment of measurement distortions in GNSS antenna array space-time processing," International Journal of Antennas and Propagation, vol. 2016, Article ID 2154763, 17 pages, 2016.

[3] A. J. O'Brien and I. J. Gupta, "Mitigation of adaptive antenna induced bias errors in GNSS receivers," IEEE Transactions on Aerospace and Electronic Systems, vol. 47, no. 1, pp. 524-538, 2011.

[4] Y. C. Chuang et al., "Prediction of antenna induced biases for GNSS receivers," in Proceedings of the International Technical Meeting of the Institute of Navigation, San Diego, CA, USA, 2014.

[5] S. K. Kalyanaraman and M. S. Braasch, "GPS adaptive array phase compensation using a software radio architecture," Journal of the Institute of Navigation, vol. 57, no. 1, pp. 53-68, 2010.

[6] U. S. Kim, D. S. De Lorenzo, D. Akos, J. Gautier, P. Enge, and J. Orr, "Precise phase calibration of a controlled reception pattern GPS antenna for JPALS," in Proceedings of the PLANS 2004 Position Location and Navigation Symposium, pp. 478-485, April 2004.

[7] D. S. De Lorenzo, Navigation Accuracy and Interference Rejection for GPS Adaptive Antenna Arrays, Stanford University, 2007. 
[8] S. Caizzone, G. Buchner, and W. Elmarissi, "Miniaturized dielectric resonator antenna array for GNSS applications," International Journal of Antennas and Propagation, vol. 2016, Article ID 2564087, 10 pages, 2016.

[9] I. Şişman and K. Yeǧin, "Reconfigurable antenna for jamming mitigation of legacy GPS receivers," International Journal of Antennas and Propagation, vol. 2017, Article ID 4563571, 7 pages, 2017.

[10] S. Backén, D. M. Akos, and M. L. Nordenvaad, "Post-processing dynamic GNSS antenna array calibration and deterministic beamforming," in Proceedings of the 21st International Technical Meeting of the Satellite Division of the Institute of Navigation, ION GNSS 2008, vol. 3, pp. 1311-1319, September 2008.

[11] C. M. Church and I. J. Gupta, "Calibration of GNSS adaptive antennas," in Proceedings of the 22nd International Technical Meeting of the Satellite Division of the Institute of Navigation 2009, ION GNSS 2009, pp. 2735-2741, 2001.

[12] S. Daneshmand, N. Sokhandan, M. Zaeri-Amirani, and G. Lachapelle, "Precise calibration of a GNSS antenna array for adaptive beamforming applications," Sensors, vol. 14, no. 6, pp. 9669-9691, 2014.

[13] A. J. O'Brien, J. Andrew, and I. J. Gupta, "Optimum adaptive filtering for GNSS antenna arrays," in Proceedings of the 21st International Technical Meeting of the Satellite Division of the Institute of Navigation, ION GNSS 2008, pp. 1301-1310, September 2008.

[14] C. L. Chang and G. S. Huang, "Low-complexity spatialtemporal filtering method via compressive sensing for interference mitigation in a GNSS receiver," International Journal of Antennas and Propagation, vol. 2014, Article ID 501025, 8 pages, 2014.

[15] G. Carrie, F. Vincent, T. Deloues, D. Pietin, and A. Renard, "A new blind adaptive antenna array for GNSS interference cancellation," in Proceedings of the 39th Asilomar Conference on Signals, Systems and Computers, pp. 1326-1330, November 2005.

[16] S. Mehmood, Z. U. Khan, F. Zaman, and B. Shoaib, "Performance analysis of the different null steering techniques in the field of adaptive beamforming," Research Journal of Applied Sciences, Engineering \& Technology, vol. 5, no. 15, pp. 4006-4012, 2013.

[17] M. D. Zoltowski and A. S. Gecan, "Advanced adaptive null steering concepts for GPS," in Proceedings of the 1995 Military Communications Conference (MILCOM). Part 1 (of 3), vol. 3, pp. 1214-1218, November 1995.

[18] A. Gecan and M. Zoltowski, "Power minimization techniques for GPS null steering antenna," in Proceedings of the 8th International Technical Meeting of the Satellite Division of The Institute of Navigation (ION GPS 1995), 1995.

[19] K. Elliott and C. Hegarty, Understanding GPS: Principles and Applications, Artech House, 2005.

[20] R. T. Compton, "The power-inversion adaptive array: concept and performance," IEEE Transactions on Aerospace and Electronic Systems, vol. 15, no. 6, pp. 803-814, 1979. 


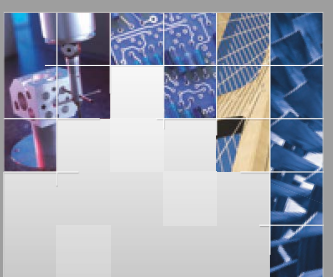

\section{Enfincering}
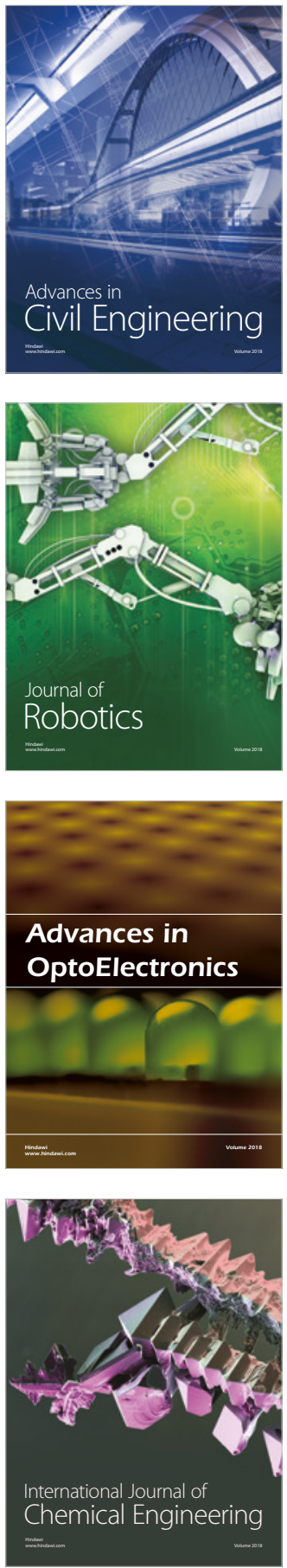

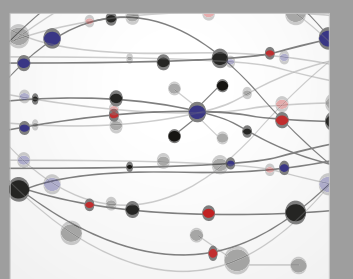

\section{Rotating \\ Machinery}

The Scientific World Journal

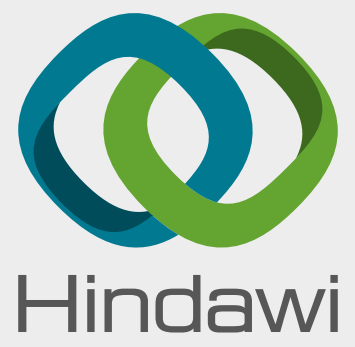

Submit your manuscripts at

www.hindawi.com
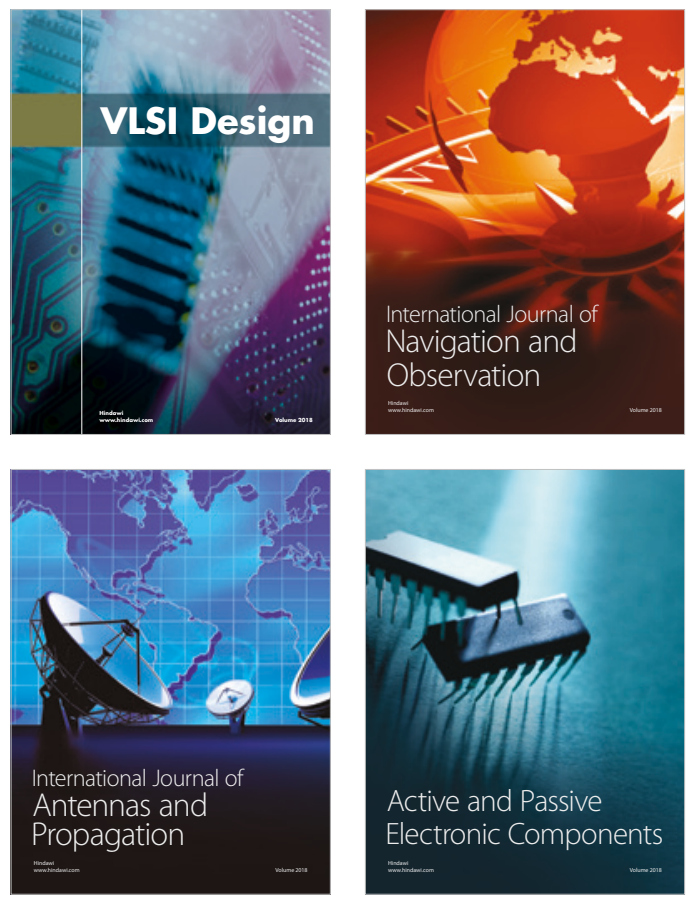
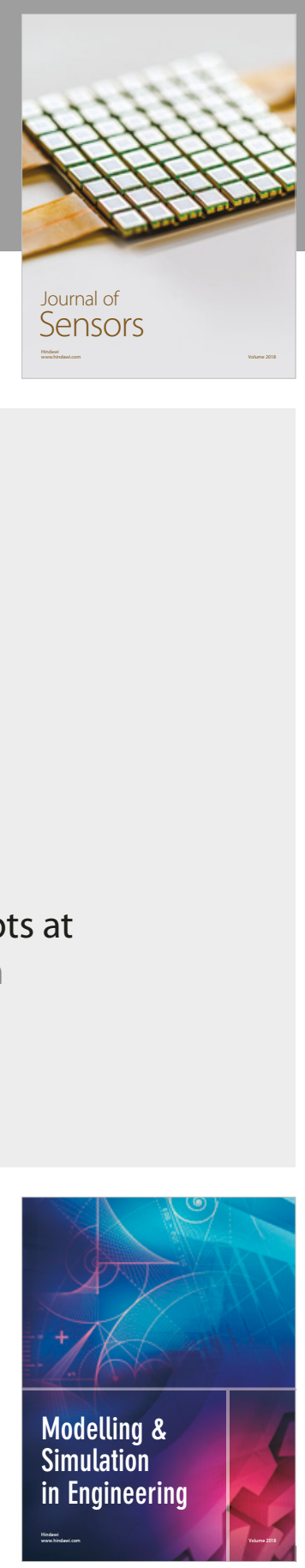

\section{Advances \\ Multimedia}
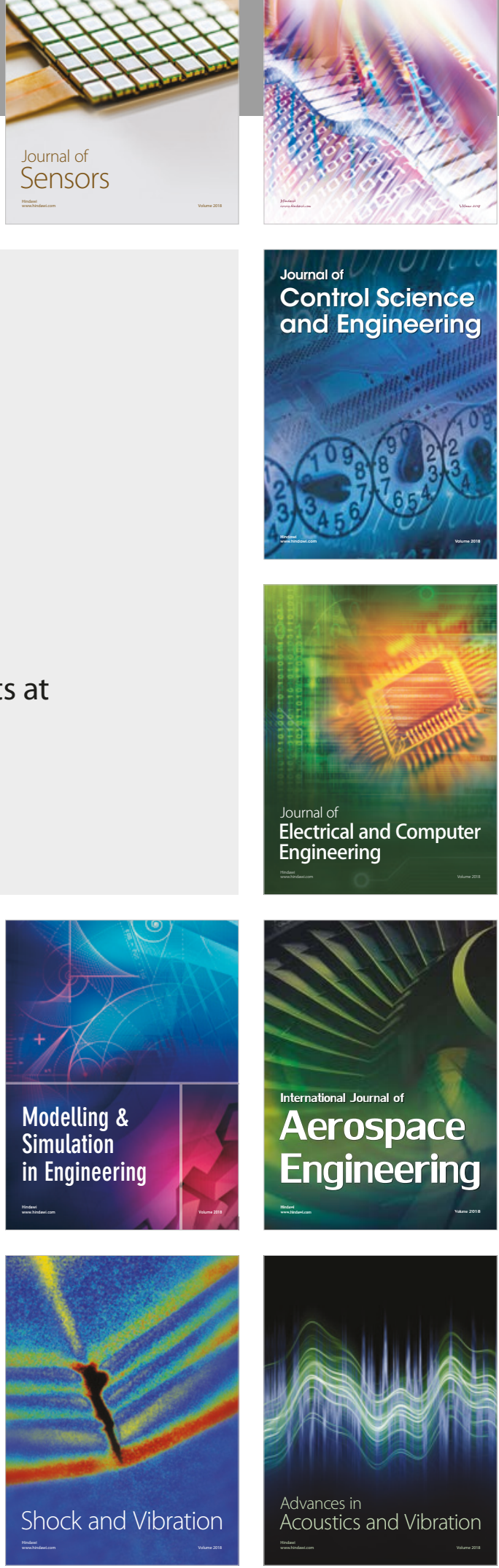